\section{Cobertura do teste de Papanicolaou e fatores associados à não-realização: um olhar sobre o Programa de Prevenção do Câncer do Colo do Útero em Pernambuco, Brasil}

\author{
Pap smear coverage and factors associated with \\ non-participation in cervical cancer screening: an \\ analysis of the Cervical Cancer Prevention Program \\ in Pernambuco State, Brazil
}

\author{
Kamila Matos de Albuquerque 1 \\ Paulo Germano Frias ${ }^{1}$ \\ Carla Lourenço Tavares de Andrade 2 \\ Estela M. L. Aquino 3 \\ Greice Menezes 3 \\ Célia Landmann Szwarcwald 4
}

\footnotetext{
${ }_{1}$ Instituto de Medicina Integral Professor Fernando Figueira, Recife, Brasil. 2 Escola Nacional de Saúde Pública Sérgio Arouca, Fundação Oswaldo Cruz, Rio de Janeiro, Brasil. 3 Instituto de Saúde Coletiva, Universidade Federal da Bahia, Salvador, Brasil. 4 Instituto de Comunicação e Informação Científica e Tecnológica em Saúde, Fundação Oswaldo Cruz, Rio de Janeiro, Brasil.

Correspondência K. M. Albuquerque Grupo de Estudo de Gestão e Avaliação em Saúde, Instituto de Medicina Integral Professor Fernando Figueira.

Rua dos Coelhos 300, Recife, PE 50070-550, Brasil. kamilamatos@imip.org.br
}

\begin{abstract}
This research aimed to assess coverage of Pap smear screening in the State of Pernambuco, Brazil, during the three years prior to the study, among women 18 to 69 years of age, and to identify factors associated with women's lack of participation in screening. This was a crosssectional, population-based study, using data from a survey in 2005-2006 with 640 women, selected by three-stage cluster sampling. Information on 258 women was analyzed. Pap smear coverage was 58.7\% for women 18 to 69 years of age and $66.2 \%$ for those 25 to 59. Single marital status, no history of childbirth, and not having consulted a physician in the previous year were associated with lack of Pap smear screening. In the multivariate analysis, low schooling also showed a significant effect. Pap smear coverage in Pernambuco was satisfactory, but insufficient to impact the epidemiological profile of cervical cancer. It is necessary to strengthen and upgrade health promotion activities in order to reduce the inequalities and encourage women's active participation in cervical cancer prevention.
\end{abstract}

Uterine Cervical Neoplasms; Vaginal Smear; Women's Health; Risk Factors

\section{Introdução}

A despeito da efetividade dos programas de rastreamento, o câncer de colo de útero ainda representa um grave problema de saúde pública, especialmente para os países em desenvolvimento que abrigam cerca de $80 \%$ dos casos e mortes decorrentes desta neoplasia 1,2 .

A infecção prévia pelo papiloma vírus humano (HPV) tem sido apontada como o principal fator de risco para o câncer de colo de útero 1,3,4,5,6. No entanto, fatores como início precoce da atividade sexual, multiplicidade de parceiros sexuais, uso de contraceptivos orais, tabagismo, situação conjugal e baixa condição sócio-econômica têm sido apontados como fatores de risco importantes para o desenvolvimento dessa neoplasia 7,8 .

Os programas de rastreamento ou screening sistemático da população feminina por meio do exame citológico do colo do útero, também conhecido como exame de Papanicolaou, têm sido uma das estratégias públicas mais efetivas, seguras e de baixo custo para detecção precoce desse câncer 9,10. Estudos indicam que mulheres que não realizam ou nunca realizaram esse exame desenvolvem a doença com maior freqüência 11,12,13 e que, em diferentes países, tem havido redução nas taxas de incidência e mortalidade por essa neoplasia após a introdução de programas de rastreamento $14,15,16,17,18$.

No Brasil, desde 2003, excetuando-se o câncer de pele (não melanoma), o câncer do colo do 
útero figura como a segunda neoplasia maligna mais comum entre as mulheres, superada apenas pelo câncer de mama ${ }^{8}$. Para o ano de 2008, são esperados 18.680 novos casos de câncer do colo do útero, o que representa uma taxa de incidência de 19 casos a cada 100 mil mulheres 19 .

A análise das taxas ajustadas de mortalidade por câncer do colo do útero no Brasil, período de 1979-2004, indica que as mesmas continuam estáveis, não tendo apresentado alterações significativas: em 1979, a taxa era de 4,97/100 mil, ao passo que, em 2004, era de 4,74/100 mil 20.

Com o objetivo de reduzir as taxas de morbimortalidade por essa neoplasia, desde 1988 o Ministério da Saúde do Brasil adota como norma a recomendação da Organização Mundial da Saúde (OMS), que propõe a realização do exame citológico do colo do útero a cada três anos, após dois exames anuais consecutivos negativos para mulheres de 25-59 anos de idade, ou que já tenham tido atividade sexual 8 . Iniciativas como o Programa Viva Mulher, lançado em 1996, e o desenvolvimento de campanhas nacionais sistemáticas têm contribuído para o aumento da cobertura do exame no país. Contudo, verificase que as taxas de incidência e de mortalidade ainda permanecem desafiando as medidas até então adotadas, sinalizando possíveis deficiências na oferta, no acesso e na qualidade das referidas ações.

Diante do exposto, reconhece-se como importante adotar estratégias que permitam a produção de dados e informações complementares sobre a cobertura do teste Papanicolaou. No Brasil, em especial nas regiões Sul e Sudeste, diversos trabalhos têm sido conduzidos nesta perspectiva. Por outro lado, na Região Nordeste, em particular no Estado de Pernambuco, Brasil, poucos estudos têm sido realizados com tal objetivo.

Considerando que o câncer de colo do útero é uma das prioridades da política de saúde do país, o quadro epidemiológico de morbimortalidade dessa neoplasia no Estado de Pernambuco e a necessidade de produzir informações mais precisas do ponto de vista das usuárias, buscou-se, no presente trabalho, avaliar a cobertura real do teste de Papanicolaou no Estado de Pernambuco, no ano de 2006, destacando os fatores associados à não-realização dele.

\section{Material e métodos}

Trata-se de um estudo transversal, de base populacional, utilizando-se os dados do inquérito da Pesquisa Mundial de Saúde: Atenção Básica, realizada no Estado de Pernambuco, no ano de 2006, sob a coordenação do Instituto de Medici- na Integral Professor Fernando Figueira (IMIP) em parceria com a Fundação Oswaldo Cruz (Fiocruz). A pesquisa foi submetida ao Comitê de Ética do IMIP e por ele aprovada.

O tamanho da amostra da Pesquisa Mundial de Saúde: Atenção Básica foi de 640 indivíduos com 18 anos ou mais de idade. Obteve-se a amostra nas informações do Censo Demográfico realizado no ano de 2000, quando o Estado de Pernambuco foi subdividido em 8.558 setores censitários.

O processo de amostragem ocorreu em três estágios. No primeiro, a amostragem foi probabilística: os setores censitários foram selecionados com probabilidade proporcional ao tamanho, dado pelo número de domicílios do setor, abrangendo as quatro regiões do estado (Metropolitana, Mata, Agreste e Sertão). Nessa etapa, pelas informações do Instituto Brasileiro de Geografia e Estatística (Censo Demográfico 2000. http:/ / www. ibge.gov.br. Agregado por setores censitários), foram considerados, primeiramente, todos os setores com domicílios particulares permanentes, isto é, aqueles que servem como residência de famílias e são construídos com material durável (permanente). Para a seleção da amostra, foram eliminados todos os setores com menos de vinte domicílios particulares permanentes.

No segundo estágio, em cada setor, foram selecionados dois pontos geográficos, de maneira aleatória. Em cada ponto geográfico, oito domicílios foram escolhidos para pesquisa, totalizando 16 domicílios por setor. Em cada domicílio, somente um residente adulto (18 anos ou mais) foi selecionado para responder ao questionário individual de acordo com a cota estabelecida por sexo e faixa etária. O responsável pelo domicílio respondeu ao questionário domiciliar.

O trabalho de campo da Pesquisa Mundial de Saúde: Atenção Básica no Estado de Pernambuco foi realizado por empresa contratada com esta finalidade. As equipes eram compostas por um supervisor e seis a oito entrevistadores, de acordo com o porte populacional do município. O treinamento das equipes foi de responsabilidade da Fiocruz e a supervisão do IMIP e da Fiocruz.

$\mathrm{O}$ instrumento utilizado neste inquérito assemelha-se ao questionário aplicado no Brasil, por ocasião de realização da Pesquisa Mundial de Saúde: Atenção Básica, inquérito nacional para avaliação do desempenho de saúde, realizada no ano de 2003 21. Porém, no ano de 2005 foi adaptado para avaliação da atenção básica em saúde, em especial, o desempenho do Programa Saúde da Família (PSF).

O questionário era modular e composto de duas partes, a domiciliar e a individual. $\mathrm{Na}$ primeira, foram investigados: composição dos 
moradores do domicílio; relação de parentesco entre os moradores; situação sócio-econômica dos moradores; informações sobre o domicílio; presença de plano de saúde privado; gastos do domicílio com assistência de saúde; cobertura e avaliação do PSF. Na segunda parte, foram abordadas: informações sócio-demográficas; autoavaliação do estado de saúde; fatores de risco; situações crônicas e comportamentos saudáveis; doenças sexualmente transmissíveis; programas de saúde, cuidados preventivos e assistenciais às crianças; assistência a idosos e avaliação da resposta do sistema de saúde do ponto de vista do usuário.

No presente estudo, foram analisados os dados contemplados nos módulos: (a) domiciliar - situação sócio-econômica e PSF e (b) individual - informações sócio-demográficas, desempenho do sistema de saúde e, particularmente, cobertura de programas de mulheres de 18-69 anos de idade.

Para avaliar a cobertura de realização de exame ginecológico com teste Papanicolaou foram incluídas na análise, de início, todas as mulheres com idade entre 18-69 anos que responderam às perguntas sobre realização dos exames preventivos. Assim, do universo de 320 mulheres, foram excluídas da análise 62. Pelo fato de o programa de prevenção de câncer de colo de útero ter foco nas mulheres de 25-59 anos de idade, a análise dos fatores associados à não-realização do teste Papanicolaou foi restrita a esse grupo etário.

Para identificar as variáveis que têm associação com o desfecho em pauta, foram utilizados modelos de regressão logística uni e multivariado. A variável dependente foi definida como "não ter realizado exame ginecológico com o teste Papanicolaou nos últimos três anos que antecederam a pesquisa", sendo estudadas as seguintes variáveis independentes:

a) Sócio-demográficas: faixa etária (18-24 anos; 25-39 anos; 40-59 anos; 60-69 anos); cor da pele (branca; não branca); situação conjugal (com companheiro; sem companheiro); ter dado a luz (sim; não);

b) Sócio-econômicas: grau de escolaridade (elementar incompleto; Ensino Fundamental incompleto; Ensino Fundamental completo ou mais); situação de trabalho (trabalha; não trabalha); número de bens $(0-5,6+)$, calculado pela soma do número bens presentes no domicílio (televisão, geladeira, aparelho de som, máquina de lavar roupa, linha de telefone fixo, linha de telefone celular, computador, forno microondas, máquina de lavar prato e carro); plano de saúde privado (sim; não);

c) Relativas aos serviços de saúde: uso regular do mesmo serviço de saúde (sim; não); tipo de serviço de saúde de uso regular (posto de saúde ou unidade de saúde da família; ambulatório de estabelecimento de saúde privado, consultório particular ou consulta domiciliar; policlínica, ambulatório de hospital público, pronto-socorro ou emergência; não costuma procurar o mesmo serviço de saúde); consulta médica no último ano (sim; não); domicílio cadastrado na unidade de saúde da família (sim; não).

Utilizou-se o aplicativo estatístico SPSS versão 13 (SPSS Inc., Chicago, Estados Unidos), para análise estatística dos dados, considerando o desenho de amostragem, que permite extrapolar os resultados para as mulheres de 18-69 anos do Estado de Pernambuco.

\section{Resultados}

Foram estudadas 258 mulheres, de 18-69 anos de idade. A Tabela 1 mostra a grande diferença nas coberturas de exame ginecológico por faixa de idade. Tanto entre as mulheres com menos de 25 anos como entre aquelas com 60-69 anos, as proporções de realização de exame ginecológico com Papanicolaou são menores que $40 \%$. Já entre as mulheres de 25-39 e 40-59 anos de idade, as coberturas de exame ginecológico nos três anos anteriores à pesquisa, são de, aproximadamente, $82 \%$ (dado não apresentado nas tabelas), decrescendo para $67 \%$ e $65 \%$, respectivamente, quando se examinam as coberturas do exame ginecológico com o teste Papanicolaou (Tabela 1).

As Tabelas 2 e 3 apresentam as proporções de realização de exame ginecológico com Papanicolaou nos últimos três anos que antecederam a entrevista entre as mulheres de 25-59 anos de idade segundo as variáveis independentes consideradas. Os maiores percentuais de não-realização do exame ginecológico com o teste Papanicolaou concentram-se entre as mulheres que

Tabela 1

Proporção de mulheres por realização de exame ginecológico com teste Papanicolaou segundo a faixa de idade. Pernambuco, Brasil, 2006.

\begin{tabular}{lccccccc}
\hline $\begin{array}{l}\text { Faixa etária } \\
\text { (anos) }\end{array}$ & $\mathbf{n}$ & Sim & $\%$ & $\mathbf{n}$ & Não & \multicolumn{2}{c}{ Total } \\
\hline $18-24$ & 13 & 32,5 & 27 & 67,5 & 40 & 100,0 \\
$25-39$ & 76 & 67,3 & 37 & 32,7 & 113 & 100,0 \\
$40-59$ & 53 & 64,6 & 29 & 35,4 & 82 & 100,0 \\
$60-69$ & 9 & 39,1 & 14 & 60,9 & 23 & 100,0 \\
Total & 151 & 58,5 & 107 & 41,5 & 258 & 100,0 \\
\hline
\end{tabular}


nunca deram à luz. Entre essas, a cobertura de exame ginecológico com preventivo foi de apenas 29\% (Tabela 2).

Diferenças foram encontradas por grau de escolaridade, embora não estatisticamente significativas, com o maior percentual de realização de exame preventivo entre as mulheres com ensino fundamental completo (71\%) e o menor entre as com ensino elementar incompleto (60\%). A proporção de realização do exame preventivo foi também mais elevada entre as participantes com maior número de bens (6 ou mais) e entre as que auto-referiram como brancas, apesar de as diferenças não terem sido estatisticamente significativas no nível de 5\%. Já a situação conjugal mostrou efeito significativo, com a maior cober- tura de exame preventivo entre as mulheres que vivem com companheiro. Nenhuma diferença foi encontrada para situação de trabalho e plano de saúde.

A análise da proporção de mulheres com exame preventivo nos três anos anteriores à entrevista, consoante variáveis relacionadas aos serviços de saúde, mostra que o maior efeito é encontrado para consulta médica nos últimos doze meses. Usar o mesmo serviço de saúde quando precisa de assistência e morar em domicílio cadastrado pelo PSF não influenciaram as coberturas (Tabela 3 ).

Os resultados da regressão logística multivariada estão apresentados na Tabela 4. Observase que ter dado à luz foi o fator mais fortemen-

Tabela 2

Proporção de realização do exame ginecológico com teste Papanicolaou entre as mulheres de 25-59 anos, segundo características sócio-demográficas e sócio-econômicas. Pernambuco, Brasil, 2006.

\begin{tabular}{|c|c|c|c|c|c|}
\hline \multirow[t]{2}{*}{ Características } & \multicolumn{2}{|c|}{ Sim } & \multirow[t]{2}{*}{ Total } & \multirow[t]{2}{*}{ Odds ratio } & \multirow[t]{2}{*}{ IC95\% } \\
\hline & $\mathbf{n}$ & $\%$ & & & \\
\hline \multicolumn{6}{|l|}{ Tem filhos } \\
\hline $\operatorname{Sim}$ & 123 & 70,7 & 174 & 0,173 & $0,049-0,610$ \\
\hline Não & 6 & 28,6 & 21 & - & - \\
\hline Total & 129 & 66,2 & 195 & & \\
\hline \multicolumn{6}{|l|}{ Grau de escolaridade } \\
\hline Até elementar incompleto & 40 & 59,7 & 67 & 1,691 & $0,818-3,498$ \\
\hline Elementar completo ao Ensino & 40 & 66,7 & 60 & 1,236 & $0,527-2,899$ \\
\hline \multicolumn{6}{|l|}{ Fundamental incompleto } \\
\hline $\begin{array}{l}\text { Ensino Fundamental } \\
\text { completo ou mais }\end{array}$ & 49 & 71,0 & 69 & - & - \\
\hline Total & 128 & 65,8 & 195 & & \\
\hline \multicolumn{6}{|l|}{ Número de bens } \\
\hline $0-5$ & 94 & 63,1 & 149 & 1,914 & $0,843-4,346$ \\
\hline $6+$ & 35 & 76,1 & 46 & - & - \\
\hline Total & 129 & 66,2 & 195 & & \\
\hline \multicolumn{6}{|l|}{ Raça/Cor } \\
\hline Branca & 38 & 70,9 & 54 & - & - \\
\hline Não branca & 86 & 66,2 & 130 & 1,248 & $0,541-2,876$ \\
\hline Total & 125 & 67,6 & 185 & & \\
\hline \multicolumn{6}{|l|}{ Situação conjugal } \\
\hline Com companheiro & 95 & 72,0 & 132 & - & - \\
\hline Sem companheiro & 34 & 54,0 & 63 & 2,224 & $1,056-4,684$ \\
\hline Total & 129 & 66,2 & 195 & & \\
\hline \multicolumn{6}{|l|}{ Situação de trabalho } \\
\hline Trabalha & 45 & 62,5 & 72 & 1,247 & $0,659-2,362$ \\
\hline Não trabalha & 84 & 68,3 & 123 & - & - \\
\hline Total & 129 & 66,2 & 195 & & \\
\hline \multicolumn{6}{|l|}{ Plano de saúde privado } \\
\hline Sim & 14 & 66,7 & 21 & - & - \\
\hline Não & 115 & 66,1 & 174 & 1,022 & $0,331-3,160$ \\
\hline Total & 129 & 66,2 & 195 & & \\
\hline
\end{tabular}


Proporção de realização do exame ginecológico com teste Papanicolaou entre as mulheres de 25-59 anos, segundo características relativas aos serviços de saúde. Pernambuco, Brasil, 2006

\begin{tabular}{|c|c|c|c|c|c|}
\hline \multirow[t]{2}{*}{ Características } & \multicolumn{2}{|c|}{ Sim } & \multirow[t]{2}{*}{ Total } & \multirow[t]{2}{*}{ Odds ratio } & \multirow[t]{2}{*}{ IC95\% } \\
\hline & $\mathbf{n}$ & $\%$ & & & \\
\hline \multicolumn{6}{|l|}{ Uso regular do mesmo serviço de saúde } \\
\hline Sim & 77 & 65,3 & 118 & - & - \\
\hline Não & 52 & 66,7 & 78 & 0,922 & $0,542-1,570$ \\
\hline Total & 128 & 65,8 & 195 & & \\
\hline \multicolumn{6}{|l|}{ Tipo de serviço de saúde de uso regular } \\
\hline Posto/PSF & 35 & 63,6 & 55 & 3,530 & $0,658-18,955$ \\
\hline $\begin{array}{l}\text { Ambulatório de estabelecimento de saúde privado } \\
\text { empresa ou sindicato; consultório }\end{array}$ & 19 & 86,4 & 22 & - & - \\
\hline \multicolumn{6}{|l|}{ Policlínica; ambulatório de estabelecimento de saúde } \\
\hline público; pronto-socorro ou emergência & 22 & 57,9 & 38 & 4,547 & $0,833-24,817$ \\
\hline Não costuma procurar o mesmo serviço de saúde & 52 & 66,7 & 78 & 3,019 & $0,576-15,833$ \\
\hline Total & 127 & 66,3 & 192 & & \\
\hline \multicolumn{6}{|l|}{ Domicílio cadastrado no PSF } \\
\hline Sim & 71 & 66,4 & 107 & - & - \\
\hline Não & 58 & 65,9 & 88 & 1,020 & $0,538-1,933$ \\
\hline Total & 129 & 66,2 & 195 & & \\
\hline \multicolumn{6}{|l|}{ Consulta ao médico no último ano } \\
\hline Sim & 121 & 68,9 & 176 & - & - \\
\hline Não & 7 & 38,9 & 18 & 3,733 & $1,362-10,231$ \\
\hline Total & 129 & 66,2 & 195 & & \\
\hline
\end{tabular}

PSF: Programa Saúde da Família.

te associado à não-realização do exame $(\mathrm{OR}=$ 0,$119 ; \mathrm{p}=0,006$ ), seguido de consulta médica no ano anterior à pesquisa $(\mathrm{OR}=4,191 ; \mathrm{p}=0,007)$. Baixo nível de escolaridade também mostrou efeito estatisticamente significativo (OR $=2,967$, $\mathrm{p}=0,029$ ) na comparação com as mulheres com Ensino Fundamental completo.

\section{Discussão}

O presente estudo objetivou levantar dados complementares sobre a cobertura do exame de Papanicolaou no Estado de Pernambuco, identificando fatores associados à não-realização dele. Considerando os aspectos metodológicos, as poucas perdas ou recusas constituíram um ponto positivo. Entretanto, por esse estudo integrar uma pesquisa-âncora, não desenhada especificamente para coletar os dados em questão, a amostra de mulheres de 25-59 anos de idade foi relativamente pequena, e perguntas importantes não puderam ser exploradas a fim de complementar a investigação com outros fatores de risco conhecidos na literatura, como início precoce da atividade sexual e multiplicidade de parceiros sexuais.

A avaliação de programas e serviços de saúde tem possibilitado o desenvolvimento de estudos com diferentes enfoques: cobertura, acesso, efetividade, eficiência, satisfação do usuário, qualidade técnico-científica entre outros. Para Vieira-da-Silva 22, avaliar a cobertura de uma intervenção constitui um pré-requisito para avaliação de outras características, já que para discutir qualidade, impacto e satisfação do usuário é preciso que as ações e serviços sejam previamente ofertados à população 22,23.

Cobertura é um termo claro, bem definido e freqüentemente descrito como a proporção da população alvo que se beneficia de determinada intervenção ${ }^{24}$. Soberón 25 faz uma distinção entre cobertura potencial e cobertura real. A primeira revela as possibilidades da oferta, enquanto a segunda, também denominada de utilização de serviços, resulta do trabalho efetivamente realizado, ou seja, corresponde à proporção da população que efetivamente se beneficiou de determinada intervenção. Para o presente estudo, a característica cobertura real foi avaliada pela 
Fatores associados à não-realização de exame ginecológico com teste Papanicolaou entre as mulheres de 25-59 anos. Pernambuco, Brasil, 2006.

\begin{tabular}{|c|c|c|}
\hline Características & Odds ratio & IC95\% \\
\hline \multicolumn{3}{|l|}{ Tem filho } \\
\hline $\operatorname{Sim}$ & 0,119 & $0,027-0,520$ \\
\hline Não & - & - \\
\hline \multicolumn{3}{|l|}{ Grau de escolaridade } \\
\hline Até elementar incompleto & 2,967 & $1,125-7,820$ \\
\hline Elementar completo ao Ensino Fundamental incompleto & 2,072 & $0,699-6,143$ \\
\hline Ensino Fundamental completo ou mais & - & - \\
\hline \multicolumn{3}{|l|}{ Plano de saúde privado } \\
\hline Sim & - & - \\
\hline Não & 0,232 & $0,061-0,873$ \\
\hline \multicolumn{3}{|c|}{ Costume de procurar o mesmo serviço de saúde quando precisa de assistência } \\
\hline Sim & - & - \\
\hline Não & 6,010 & $1,051-34,364$ \\
\hline \multicolumn{3}{|l|}{ Costume de procurar o mesmo serviço de saúde } \\
\hline Posto/Programa Saúde da Família & 7,059 & $1,153-43,222$ \\
\hline $\begin{array}{l}\text { Ambulatório de estabelecimento de saúde privado, } \\
\text { empresa ou sindicato; consultório }\end{array}$ & - & - \\
\hline $\begin{array}{l}\text { Policlínica; ambulatório de estabelecimento de saúde público; } \\
\text { pronto-socorro ou emergência }\end{array}$ & 8,501 & $1,289-56,058$ \\
\hline Não costuma procurar o mesmo serviço de saúde & 1,000 & $1,000-1,000$ \\
\hline \multicolumn{3}{|l|}{ Consulta ao médico } \\
\hline Sim & - & - \\
\hline Não & 4,191 & $1,520-11,552$ \\
\hline
\end{tabular}

perspectiva de utilização do serviço de prevenção do câncer do colo do útero, julgado pela proporção de mulheres de 25-59 anos que realizaram o exame ginecológico com o teste Papanicolaou nos três anos que antecederam a entrevista.

Os principais resultados apontam para uma cobertura do teste Papanicolaou no Estado de Pernambuco maior do que $60 \%$ entre as mulheres de 25-59 anos de idade; uma expressiva concentração de mulheres sem filhos, porém em idade reprodutiva que se encontram descobertas pelo exame preventivo; e a presença de desigualdades sociais na realização do exame Papanicolaou, embora não muito acentuadas pelos limites da amostra estudada.

Dentre as recomendações da Organização Mundial de Saúde para impactar o perfil epidemiológico do câncer do colo do útero tem-se de: garantir uma cobertura de rastreamento mínima de $80 \%$ a $85 \%$ da população feminina de 25 59 anos, prezar pela qualidade de realização do exame citológico e garantir tratamento oportuno e seguimento das pacientes 26 . Dificuldades relatadas pelos países em desenvolvimento para impactar nas taxas de incidência e mortalidade por câncer do colo do útero, freqüentemente, guardam associação com a não-garantia desses fatores 27 .

Os resultados deste estudo sugerem que uma expressiva parcela das mulheres pernambucanas, entre 25-59 anos de idade, população-alvo do programa de prevenção do câncer do colo do útero, realizou o teste Papanicolaou com intervalo de tempo máximo recomendado pelo Ministério da Saúde do Brasil. Por outro lado, o diferencial entre a proporção de mulheres com cobertura de exame ginecológico nos três anos anteriores à pesquisa (aproximadamente 82\%) e os percentuais mais baixos de coberturas do exame ginecológico com teste de Papanicolaou sugere oportunidades perdidas de rastreamento da população feminina que seriam importantes para melhorar a situação evidenciada ainda aquém do preconizado pela OMS para impactar o perfil epidemiológico do câncer do colo do útero.

Os resultados deste estudo guardam semelhança com os resultados do Inquérito Populacional Nacional, realizado no ano de 2003, que 
encontrou cobertura de $65,5 \%$ para mulheres de 18-69 anos 28 . Por outro lado, os mesmos se encontram aquém dos resultados publicados a partir do inquérito nacional desenvolvido em 15 capitais brasileiras no período 2002-2003, que encontrou coberturas entre $73 \%$ e $92 \%$ para mulheres entre 25-69 anos 29 e do estudo de base populacional realizado na cidade de Pelotas, Rio Grande do Sul, Brasil, que encontrou cobertura de $72 \%$ para mulheres entre $20-69$ anos 30 . Todavia, tais diferenças de cobertura podem guardar relação com o fato de ambos os estudos terem sido realizados com intervalo de faixa etária maior e nas zonas urbanas onde, em geral, há maior disponibilidade de serviços, bens e atividades que facilitam a realização do exame preventivo para o câncer do colo do útero.

Mulheres pertencentes às faixas etárias mais jovens e as com mais idade foram as que menos realizaram o exame ginecológico com o Papanicolaou nos últimos três anos, concordando com o observado por outros autores 31,32,33. Este resultado nos remete à reflexão sobre a insuficiência da ampliação da oferta do referido procedimento no Sistema Único de Saúde, quando descontextualizada de uma estratégia para promoção da saúde focada na intersetorialidade. A ênfase na promoção da saúde no Programa de Controle do Câncer do Colo do Útero pode ser decisiva na ampliação da cobertura do teste de Papanicolaou entre as mulheres mais facilmente suscetíveis ao agravo.

A despeito das variações de cobertura encontradas, verifica-se que a maioria dos estudos citados possui um achado em comum: mulheres que mais poderiam se beneficiar do teste de Papanicolaou são as que menos o realizam; o que pode, em parte, explicar o diagnóstico tardio e a manutenção das taxas de mortalidade.

No nosso estudo, particularmente, as mulheres que têm ensino elementar incompleto foram as que mostraram a menor cobertura, corroborando com demais estudos 30,31,32,33,34,35,36. Além da baixa escolaridade, bem documentada na literatura científica como um fator de risco para o desenvolvimento do câncer do colo do útero $7,8,36$, destacam-se os achados relativos às variáveis dar à luz e situação conjugal, corroborando com os resultados descritos por outros autores $31,32,36,37,38$, sendo a condição de não ter filho o principal fator associado à não-realização do exame preventivo. Em geral, a realização do preventivo acontece em conjunção às atividades de rotina da assistência ginecológica, obstétrica ou de planejamento familiar. Este resultado indica a necessidade de integralizar a atenção à saúde da mulher pela ampliação da oferta do teste Papanicolaou para além de um procedimento de rotina ofertado durante as consultas ginecológicas e de pré-natal, objetivando estender seus benefícios para toda mulher, independentemente de sua experiência maternal e da sua situação conjugal.

Outro achado merecedor de atenção foi o fato de que morar em domicílio cadastrado pelo PSF não influenciou a cobertura do exame ginecológico com teste Papanicolaou com intervalo de tempo máximo recomendado pelo Ministério da Saúde do Brasil. Considerando a importância da Estratégia Saúde da Família na reorganização da atenção básica, a premissa de funcionar como porta de entrada para o sistema público de saúde e a cobertura dessa estratégia no Estado de Pernambuco (70\%) é recomendável que os gestores intervenham mediante processos sistemáticos de monitoramento e avaliação visando à melhoria da atenção à saúde prestada.

Em razão do acelerado processo de expansão do PSF vivenciado por diversos municípios, inclusive os pernambucanos, ênfase deve ser dada ao fortalecimento e qualificação das ações de promoção da saúde, no âmbito da atenção básica, na perspectiva de construir uma agenda integrada e participativa que busque reduzir as situações de desigualdade e estimular o protagonismo das mulheres nas ações para prevenção do câncer de colo de útero 39 . 


\section{Resumo}

Buscou-se avaliar a cobertura do teste Papanicolaou no Estado de Pernambuco, Brasil, nos três anos anteriores à pesquisa, entre mulheres de 18-69 anos, e identificar fatores associados à sua não-realização. Trata-se de um estudo transversal, de base populacional, utilizando-se dados de inquérito realizado no período 2005-2006 com 640 indivíduos, selecionados por amostragem por conglomerados em três estágios de seleção. Foram analisadas informações sobre $258 \mathrm{mu}$ lheres. A cobertura do Papanicolaou entre mulheres de 18-69 anos foi de 58,7\% e de 25-59 anos de 66,2\%. Viver sem companheiro, não ter dado à luz e não ter realizado consulta médica no último ano mostraram associação com a não-realização do teste. Na análise multivariada, o baixo grau de escolaridade mostrou também efeito significativo. A cobertura do Papanicolaou em Pernambuco foi satisfatória, porém insuficiente para impactar no perfil epidemiológico do câncer do colo uterino. É preciso fortalecer e qualificar as ações de promoção da saúde, visando reduzir as desigualdades e estimular o protagonismo das mulheres nas ações de prevenção do câncer do colo uterino.

Neoplasias do Colo do Útero; Esfregaço Vaginal; Saúde da Mulher; Fatores de Risco

\section{Referências}

1. Parkin DM, Bray F, Ferlay J, Pisani P. Global cancer statistics, 2002. CA Cancer J Clin 2005; 55:74-108.

2. Fonseca LAM, Ramacciotti AS, Eluf Neto J. Tendência da mortalidade por câncer de útero no município de São Paulo entre 1980 e 1999. Cad Saúde Pública 2004; 20:136-42.

3. Trottier H, Franco EL. Human papillomavirus and cervical cancer: burden of illness and basis for prevention. Am J Manag Care 2006; 12 Suppl 1:462-72.

4. Bosch FX, Lorincz A, Muñoz N, Meijaer CJLM, Shah KV. The causal relation between human papillomavirus and cervical cancer. J Clin Pathol 2002; 55:244-65.

5. Walboomers JMM, Jacobs MV, Mannos MM, Bosch XF, Kummer A, Shah KV, et al. Human papillomavirus, a necessary cause of invasive cervical cancer wordwide. J Pathol 1999; 189:12-9.

6. Rama CH, Roteli-Martins CM, Derchain SFM, Oliveira EZ, Aldrighi JM, Mariane-Neto C. Detecção sorológica de anti-HPV 16 e 18 e sua associação com os achados do Papanicolaou em adolescentes e mulheres jovens. Rev Assoc Med Bras (1992) 2006; 52:43-7.

\section{Colaboradores}

K. M. Albuquerque, P. G. Frias e C. L. T. Andrade elaboraram o projeto de pesquisa, participaram da coleta e análise dos dados e da redação do artigo. E. M. L. Aquino, G. Menezes participaram na elaboração do projeto, revisão dos dados e da redação do artigo. C. L. Szwarcwald revisou a análise dos dados e a redação do artigo.

\section{Agradecimentos}

Este trabalho teve auxílio financeiro da Fundação Ford, por intermédio do Programa de Introdução à Metodologia de Pesquisa em Gênero, Sexualidade e Saúde Reprodutiva (Núcleo de Estudos de População - Universidade Estadual de Campinas; Instituto de Medicina Social - Universidade do Estado do Rio de Janeiro; Instituto de Saúde Coletiva - Universidade Federal da Bahia) e da Pesquisa Mundial de Saúde: Atenção Básica, realizada no Estado de Pernambuco, do qual este estudo é tributário.
7. Castro-Jiménez MA, Vera-Cala LM, Posso-Valencia HJ. Epidemiología del cáncer de cuello uterino: estado del arte. Rev Colomb Obstet Ginecol 2006; 57:182-9.

8. Departamento de Atenção Básica, Secretaria de Atenção à Saúde, Ministério da Saúde. Controle dos cânceres do colo do útero e de mama. Brasília: Ministério da Saúde; 2006.

9. Solomon D, Breen N, McNeel T. Cervical cancer screening rates in the United States and the potential impact of. Implementation of screening guidelines CA Cancer J Clin 2007; 57:105-11.

10. Caetano R, Vianna CMM, Thuler, LCS, Girianelli VR. Custo-efetividade no diagnóstico precoce do câncer de colo uterino no Brasil. Physis (Rio J.) 2006; 16:99-118.

11. Pinho AA, França-Júnior I. Prevenção do câncer de colo do útero: um modelo teórico para analisar o acesso e a utilização do teste de Papanicolaou. Rev Bras Saúde Matern Infant 2003: 3:95-112.

12. Herrero R, Brinton LA, Reeves WC, Brenes MM, Tenorio F, Britton RC, et al. Factores de riesgo de carcinoma invasor del cuello uterino en América Latina. Bol Oficina Sanit Panam 1990; 109:6-26. 
13. Eluf Neto J, Nascimento CM. Cervical cancer in Latin America. Semin Oncol 2001; 28:188-97.

14. Baker D, Middleton E. Cervical screening and health inequality in England in the 1990s. J Epidemiol Community Health 2003; 57:417-23.

15. Anttila A, Nieminen P. Cervical cancer screening programme in Finland. Eur J Cancer 2000; 36:2209-14.

16. Segnan N, Ronco G, Ciatto S. Cervical cancer screening in Italy. Eur J Cancer 2000; 36:2235-9.

17. Gustafsson L, Ponten J, Zack M, Adami HO. International incidence rates of invasive cervical cancer after introduction of cytological screening. Cancer Causes Control 1997; 8:755-63.

18. Hakama M, Potential contribution of screening to cancer mortality reduction. Cancer Detect Prev 1993; 17:513-20.

19. Instituto Nacional de Câncer. Incidência 2008: estimativas de câncer no Brasil. http:/ /www.inca.gov. br/estimativa/2007/ (acessado em 10/Dez/2007).

20. Martins LFL, Thuler LCS, Valente JG. Cobertura do exame de Papanicolaou no Brasil e seus fatores determinantes: uma revisão sistemática da literatura. Rev Bras Ginecol Obstet 2005; 27:485-92.

21. Szwarcwald CL, Viacava F, Vasconcellos MTL, Leal MC, Azevedo LO, Queiroz RSB, et al. Pesquisa Mundial de Saúde 2003: o Brasil em números. RADIS Comunicação em Saúde 2004; 23:14-33.

22. Vieira-da-Silva LM, Hartz ZMA, organizadoras. Avaliação em saúde: dos modelos teóricos à prática na avaliação de programas e sistemas de saúde. Rio de Janeiro: Editora Fiocruz/Salvador: Edufba; 2005.

23. Vieira-da-Silva LM, Formigli VLA, Cerqueira MP, Kruchevsky L, Nascimento CL. O processo de distritalização e a utilização de serviços de saúde - avaliação do caso de Pau da Lima. Cad Saúde Pública 1995; 11:72-84.

24. Rossi PH, Freeman HE. Evaluation, a systematic approach. Beverly Hills: Sage Publications; 2004.

25. Soberón G. La extensión de cobertura de los servicios de salud. Gac Med Mex 1988; 124:163-6.

26. World Health Organization. Manual on the prevention and control of common cancers. Geneva: World Health Organization; 1998. (Westerns Pacific Series, 20).

27. Suba EJ, Donnelly AD, Furia LM, Huynh ML, Raab SS. Cervical cancer prevention for all the world's women: genuine promise resides in skilled quality management rather than novel screening approaches. Diagn Cytopathol 2007; 35:187-91.
28. Szwarcwald CL, Leal MC, Gouveia GC, Souza, WV. Desigualdades socioeconômicas em saúde no Brasil: resultados da Pesquisa Mundial de Saúde, 2003. Rev Bras Saúde Matern Infant 2005; 5 Suppl: S1-22.

29. Instituto Nacional de Câncer. Inquérito domiciliar sobre comportamentos de risco e morbidade referida de doenças e agravos não transmissíveis. Rio de Janeiro: Instituto Nacional de Câncer; 2004.

30. Dias-da-Costa JS, Olinto MTA, Gigante DP, Menezes AMB, Macedo S, Borba AT, et al. Cobertura do exame citopatológico na cidade de Pelotas, Rio Grande do Sul, Brasil. Cad Saúde Pública 2003; 19:191-7.

31. Nascimento CM, Eluf Neto J, Rego RA. Pap test coverage in São Paulo municipality and characteristics of the women tested. Bull Pan Am Health Organ 1996; 30:302-12.

32. Hackenhaar AA, Cesar JA, Domingues MR. Exame citopatológico de colo uterino em mulheres com idade entre 20 e 59 anos em Pelotas, RS: prevalência, foco e fatores associados à sua não-realização. Rev Bras Epidemiol 2006; 9:103-11.

33. Oliveira MMHN, Silva AAM, Brito LMO, Coimbra LC. Cobertura e fatores associados à não-realização do exame preventivo de Papanicolaou em São Luís, Maranhão. Rev Bras Epidemiol 2006; 9:325-34.

34. Quadros CAT, Victora CG, Dias-da-Costa JS. Coverage and focus of a cervical cancer prevention program in Southern Brazil. Rev Panam Salud Pública 2004; 16:223-38.

35. Amorim VMSL, Barros MBA, Cesar CLG, Carandina L, Goldbaum M. Fatores associados à não-realização do exame de Papanicolaou: um estudo de base populacional no Município de Campinas, São Paulo, Brasil. Cad Saúde Pública 2006; 22:2329-38.

36. Cesar JA, Horta BL, Gomes G, Houlthusen RS, Willrich RM, Kaercher A, et al. Fatores associados à não-realização de exame citopatológico de colo uterino no extremo Sul do Brasil. Cad Saúde Pública $2003 ; 19: 1365-72$.

37. Novaes HMD, Braga PE, Schout D. Fatores associados à realização de exames preventivos para câncer nas mulheres brasileiras, PNAD 2003. Ciênc Saúde Coletiva 2006; 11:1023-35.

38. Leal MC, Gama SGN, Frias P, Szwarcwald CL. Healthy lifestyles and access to periodic health exams among Brazilian women. Cad Saúde Pública 2005; 21 Suppl 1:S78-88.

39. Ministério da Saúde. Política Nacional de Promoção da Saúde. http://bvsms.saude.gov.br/bvs/ publicacoes/portaria687_2006_anexo1.pdf (acessado em 29/Jan/2007).

Recebido em 26/Fev/2008

Versão final reapresentada em 19/Dez/2008

Aprovado em 05/Mar/2009 